Open Access : ISSN : 1848-7718

Original scientific paper

http://www.pub.iapchem.org/ojs/index.php/admet/index

\title{
Characterization of SLC transporters in human skin
}

\author{
Marion Alriquet, Karine Sevin, Alexandre Gaborit, Pierre Comby, Bernard Ruty and \\ Hanan Osman-Ponchet ${ }^{*}$
}

Galderma R\&D, les Templiers, 2400 route des Colles, BP 87, F-06902 Sophia-Antipolis

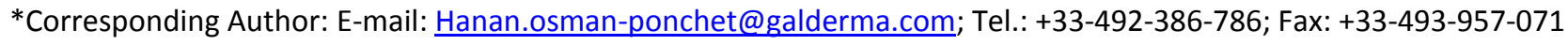

Received: January 30, 2015; Revised: March 19, 2015; Published: March 31, 2015

\begin{abstract}
Most identified drug transporters belong to the ATP-binding Cassette (ABC) and Solute Carrier (SLC) families. Recent research indicates that some of these transporters play an important role in the absorption, distribution and excretion of drugs, and are involved in clinically relevant drug-drug interactions for systemic drugs. However, very little is known about the role of drug transporters in human skin in the disposition of topically applied drugs and their involvement in drug-drug interactions. The aim of this work was to compare the expression in human skin (vs human hepatocytes and kidney) of SLC transporters included in the EMA guidance as the most likely clinical sources of drug interactions. The expression of SLC transporters in human tissues was analyzed by quantitative RT-PCR. Modulation of SLC47A1 and SLC47A2 (MATE1 and MATE2) expression was analyzed after treatment of human skin in organ-culture with rifampicin and UV irradiation. The expression of SLCO2B1 (OATPB), SLCO3A1 (OATPD), SLCO4A1 (OATPE), SLC47A1 and SLC4TA2 (MATE1 and MATE2) was detected in human skin, OATPE and MATE1 being the most expressed. OATPE is about 70 times more expressed in human skin than in human hepatocytes. Moreover, the expression of SLC47A1 and SLC47A2 was down-regulated after treatment with rifampicin or after exposure to UV light. The present findings demonstrate that SLCO4A1 (OATPE) and SLC47A1 (MATE1) are highly expressed in human skin and suggest the involvement of SLC transporters in the disposition of topically applied drugs.
\end{abstract}

\section{Keywords}

Solute-carrier transporter; MATE1; UV light; Skin absorption

\section{Introduction}

The human skin, one of the largest organ in the organism with about $6 \%$ of total body weight (5-6 kg, $2 \mathrm{~m}^{2}$ in area), acts as a physical and biochemical barrier to protect the body from environmental factors and also plays a pivotal role in homeostasis by preventing the uncontrolled loss of water [1]. Although constitutive expression of xenobiotic-metabolizing enzymes has been detected in normal keratinocytes $[2,3]$ the levels of drug-metabolizing enzymes are generally much lower in the skin than those in the liver and intestine [4-7]. Thus, skin permeability rather than drug metabolism appears to be the major barrier to topical bioavailability $[8,9]$. 
Most identified drug transporters belong to the ATP-binding Cassette (ABC) and Solute Carrier (SLC) families. Xenobiotic transporters ( $A B C S$ and SLCS) have broad specificity and are involved in both uptake (influx) and secretion (efflux) of their substrates, thereby affecting their cellular disposition. Constitutive expression of multidrug-resistance associated proteins (MRP, $A B C C$ ) or solute carrier organic anion transporting polypeptides (OATPs) has been detected in human keratinocytes $[2,3,10,11]$. The expression of $A B C B 1$ (multidrug resistance protein 1, P-glycoprotein or P-gp) has only been found after keratinocyte treatment with dexamethasone [2].

Current knowledge about the function of xenobiotic transporters in the skin is limited. In addition to their role as a contributor to multidrug resistance, it has been demonstrated that P-gp as well as MRP1 help maintain a healthy immune response in the skin as they play an important role in the migration of Langerhans cells out of the skin by way of the lymphatic vessels [12]. In addition, these transporters also play a role in the active efflux of contact allergens and endogenous compounds such as steroid hormones from normal human epidermal keratinocytes (NHEK) [13]. Further studies demonstrated P-gp involvement in migration and invasion of resistant melanoma cells [14], whereas very low or no expression of P-gp was detected in primary human melanocytes [15]. Recently the expression pattern of human ATP-binding cassette transporters was reported in human skin [16]. In addition the characterization of $A B C$ transporters in human skin was reported recently by our group [17].

The present study was not intended to be an exhaustive search for SLC transporter expression in human skin. This has been reported in human keratinocytes by Schiffer et al. [3] and in human skin by Li et al. [18] and more recently by Fujiwara et al. [19]. It should be noted that MATE1 and MATE2 transporters were not included in those studies. The aim of our study was to compare the expression in human skin (vs human hepatocytes and kidney) of SLC transporters included in the EMA guidance [20] as the most likely clinical sources of drug interactions (SLCO1B1, SLCO1B3, SLC22A1, SLC22A2, SLC22A6, SLC22A8, SLC47A1 and SLC47A2). This list of SLC transporters is essentially the same as the one reported in the recent review by Maeda and Sugiyama [21]: list which is based on the FDA guidance [22] to which these authors added MATE1 and MATE2 transporters.

Three others transporters SLCO2B1 (OATPB), SLCO3A1 (OATPD) and SLCO4A1 (OATPE) were included as a control as they are known to be expressed in human skin $[3,18,19]$. Furthermore, the modulation of expression of SLC47A1 and SLC47A2 (MATE family) transporters by rifampicin and UV light exposure was also evaluated in human skin in organ-culture.

\section{Experimental}

\section{Chemicals and reagents}

Rifampicin was from Sigma-Aldrich (St Louis, MO, USA). Total RNA from human kidneys came from Life Technologies (Carlsbad, CA, USA).

\section{Skin organ-culture}

Fresh healthy human skin surgical waste samples excised during cosmetic surgery were collected and used with the consent of the patients. Skin tissues were handled under aseptic condition and processed with sterile dissection tools. After removing adipose tissue and hypodermis with surgical scissors, skin biopsies of $6 \mathrm{~mm}$ diameter were taken using sterile biopsy punch tools, and transferred to a 6-well plate 
filled with long term skin culture medium (Biopredic, France). This proprietary long-term skin culture medium has been developed specifically to maintain the survival of human skin explants. The specific composition of this medium (fetal calf serum, antibiotics, pituitary bovine extract, sodium pyruvate, growth factors and hormones such as insulin and epidermal growth factor, hydrocortisone and selenium) allows the preservation of dermal and epidermal cell populations in normal metabolic conditions for at least 3 days. Four biopsies per well were used (Figure 1). The culture plate was maintained in a humidified incubator at $37^{\circ} \mathrm{C}$ with $5 \% \mathrm{CO}_{2}$.

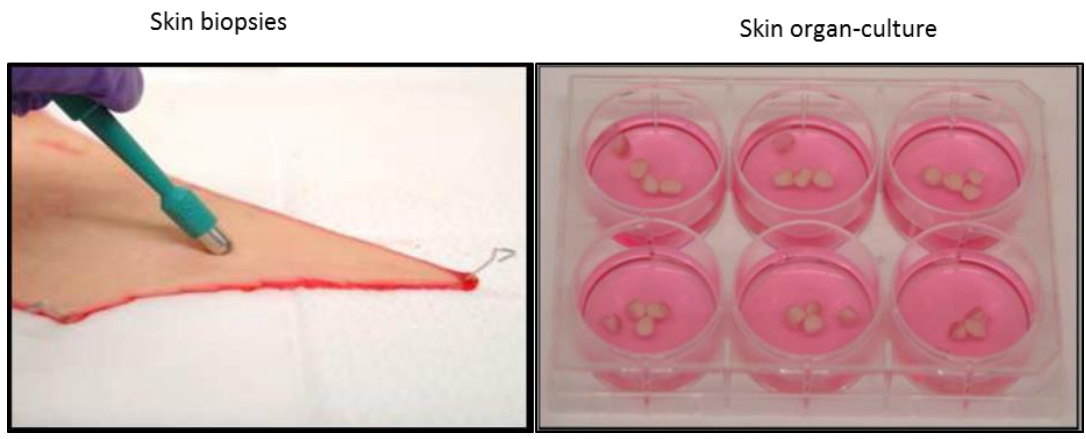

Figure 1. Skin organ-culture. Skin biopsies were taken using biopsy punch and transferred to a 6-well plate filled with culture medium.

\section{Treatment with rifampicin}

Skin biopsies were treated during 72 hours with $20 \mu \mathrm{M}$ rifampicin, refreshing culture medium every 24 hours. Control skin biopsies were cultured in the same conditions but rifampicin was omitted in the vehicle (i.e. $0.1 \% \mathrm{DMSO}$ ). During incubation, culture plate was placed in a humidified incubator at $37{ }^{\circ} \mathrm{C}$ with $5 \%$ $\mathrm{CO}_{2}$. Skins from at least three different individuals were used in each experiment unless mentioned.

\section{Treatment with sunlight simulator}

Skin biopsies were exposed during one hour every day during 3 days in a sunlight simulator (Model 91293; Oriel Instruments) equipped with a 1000-W Xenon arc lamp power supply set at $850 \mathrm{~W}$. The sunlight simulator dispensed a mix of UVA at $110 \mathrm{~W} / \mathrm{m}^{2}$ and UVB at $20 \mathrm{~W} / \mathrm{m}^{2}$ which corresponds to approximately $40 \mathrm{~J} / \mathrm{cm}^{2}$ UVA and $3.6 \mathrm{~J} / \mathrm{cm}^{2}$ UVB for a 1 hour exposure period. Control skin biopsies were cultured in the same conditions but without exposure in the sunlight simulator.

\section{Human hepatocytes in primary culture}

Fresh human hepatocytes in primary culture were purchased from Biopredic (France). Hepatocytes (identification number HH0711B) were seeded at $1.8 \times 10^{6}$ cells/well in 6-well plate. Each well was filled with $2 \mathrm{~mL}$ SPFM culture medium (Gibco, France). Hepatocyte culture was maintained during 3 days in a humidified incubator set at $37{ }^{\circ} \mathrm{C}$ with $5 \% \mathrm{CO}_{2}$. Culture medium was refreshed every day. In some experiments, hepatocytes were treated with $20 \mu \mathrm{M}$ rifampicin during 72 hours with refreshing culture medium every 24 hours.

\section{Cryopreserved human hepatocytes}

Cryopreserved human hepatocytes were used to evaluate constitutive expression of SLC transporters. Cryopreserved human hepatocytes, batch number HUE26C from Life Technologies, consisted of a pool of 26 different donors. 


\section{Total RNA isolation}

After homogenization of skin samples or hepatocytes in lysis buffer (Promega, France), total RNA was isolated using SV Total RNA Isolation System (Promega, France) according to the instructions provided by the supplier. RNA concentrations were quantified at $260 \mathrm{~nm}$ using Biotek Synergy 2 Multi-Mode Microplate.

\section{Human kidney total RNA}

Human kidney total RNA samples were purchased from Life Technologies. Two different samples of total RNA from two women were provided at final concentration $1 \mathrm{mg} / \mathrm{mL}$ in $1 \mathrm{mM}$ sodium citrate, $\mathrm{pH}$ 6.4. RNA samples were stored at $-80^{\circ} \mathrm{C}$.

\section{Real time PCR}

Expression of eleven human SLC transporters (SLCO1B1, SLCO1B3, SLCO2B1, SLCO3A1, SLCO4A1, SLC22A1, SLC22A2, SLC22A6, SLC22A8, SLC47A1 and SLC47A2) and 4 nuclear receptors (AHR, NR113, NR112 and RXRA) was measured by real-time PCR techniques. Experiments were carried out on a 7500 Real-Time PCR System (Applied Biosystems) using TaqMan Assay-on-Demand gene expression products. For this, 500 ng of total RNA were reverse-transcribed using the High Capacity RNA to cDNA Master Mix kit (Applied Biosystems).

Table 1. Assay-on-demand used in the gene expression profile experiments.

\begin{tabular}{|c|c|}
\hline Gene/Protein & Assay-On-Demand reference \\
\hline SLCO1B1/OATP1B1 & Hs00272374_m1 \\
\hline SLCO1B3/OATP1B3 & Hs00251986_m1 \\
\hline SLCO2B1 (SLC21A9)/OATPB & Hs00939778_m1 \\
\hline SLCO3A1 (SLC21A11)/OATPD & Hs00983988_m1 \\
\hline SLCO4A1 (SLC21A12)/OATPE & Hs01030343_m1 \\
\hline SLC22A1/OCT1 & Hs00427552_m1 \\
\hline SLC22A2/OCT2 & Hs01010723_m1 \\
\hline SLC22A6/OAT1 & Hs00537914_m1 \\
\hline SLC22A8/OAT3 & Hs00188599_m1 \\
\hline SLC47A1/MATE1 & Hs00217320_m1 \\
\hline SLC47A2/MATE2 & Hs00945650_m1 \\
\hline $\mathrm{AHR} / \mathrm{AhR}$ & Hs00169233_m1 \\
\hline NR1I3/CAR & Hs00231959_m1 \\
\hline NR1I2/PXR & Hs01114267_m1 \\
\hline $\mathrm{RXRA} / \mathrm{RXR} \alpha$ & Hs01067636_m1 \\
\hline GAPDH & Hs99999905_m1 \\
\hline
\end{tabular}


PCR amplifications were performed in a total volume of $25 \mu \mathrm{L}$ using the TaqMan Universal Master Mix (Applied Biosystems). Denaturation was performed at $95{ }^{\circ} \mathrm{C}$ for $10 \mathrm{~min}$, followed by $40 \mathrm{PCR}$ cycles with the following specifications: $95^{\circ} \mathrm{C} 15 \mathrm{~s}$ and $60^{\circ} \mathrm{C} 60 \mathrm{~s}$.

Glyceraldehyde-3-phosphate dehydrogenase (GAPDH) was used as a reference gene for normalization in each sample. The references of primer and TaqMan probe sequences used in the expression profiling experiments are indicated in Table 1. All real-time PCR measurements were performed in triplicate. The comparative threshold cycle $(\mathrm{Ct})$ method, also called delta $\mathrm{Ct}$ method was used to analyse the real time PCR data.

\section{Statistical analysis}

All experiments were performed at least three times. Data are expressed as mean \pm SEM. Student's t-test was used to determine the statistical significance of differences between two groups. A $p$ value of less than 0.05 was considered as statistically significant.

\section{Results}

\section{Gene expression of SLC transporters in human skin}

The expression of 11 SLC transporters (8 of clinically importance in drug-drug interactions and three controls) was measured in human skin (three different donors) by Real Time PCR (Figure 2A). Among the 11 tested transporters, the expression of six SLC transporters was not detected in human skin (OATP1B1, OATP1B3, OCT1, OCT2, OAT1 and OAT3) while the expression of 5 SLC transporters was observed. OATPB, OATPD and MATE2 were found to be poorly expressed while two SLC transporters OATPE and MATE1 were found to be highly expressed. Data from our laboratory showed that expression level of MATE1 transporters gene was not markedly modified in cultured skin samples (Ct 26.6) compared to tissues without culture (Ct 25.1) (data not shown). These results show that some of SLC transporter involved in clinically relevant drug-drug interactions for systemic drugs are expressed in human skin and that human skin in organ-culture is a useful tool to study the involvement of SLC transporters in the absorption and distribution of topically applied drugs.

\section{Comparison of expression of SLC transporters in human skin, hepatocytes and kidney}

Liver and kidney tissues were used as a positive control because strong expression of SLC transporters has been described previously in these organs [23]. The results show that the expression profiles of SLC transporters are specific to the organ considered. In human cryopreserved hepatocytes OCT1 and OATP1B1 transporters are the most strongly expressed (Figure 2B; middle) while in human kidney (Figure 2C; bottom), it is OAT1 and OAT3. Moreover, OATPE expression in human skin is 70 times higher than in human hepatocytes and as high as in human kidney.

\section{Effect of rifampicin on gene expression of SLC transporters in human skin}

Previous studies showed that treatment with rifampicin decreased the expression level of $A B C C 1$ (MRP1) in human skin [17] and in HepG2 cells [24]. The effect of rifampicin on the expression of SLC transporters was thus investigated. After 72 hours treatment of skin samples with rifampicin $20 \mu \mathrm{M}$, gene expression of SLC transporters was measured by Real Time PCR. 


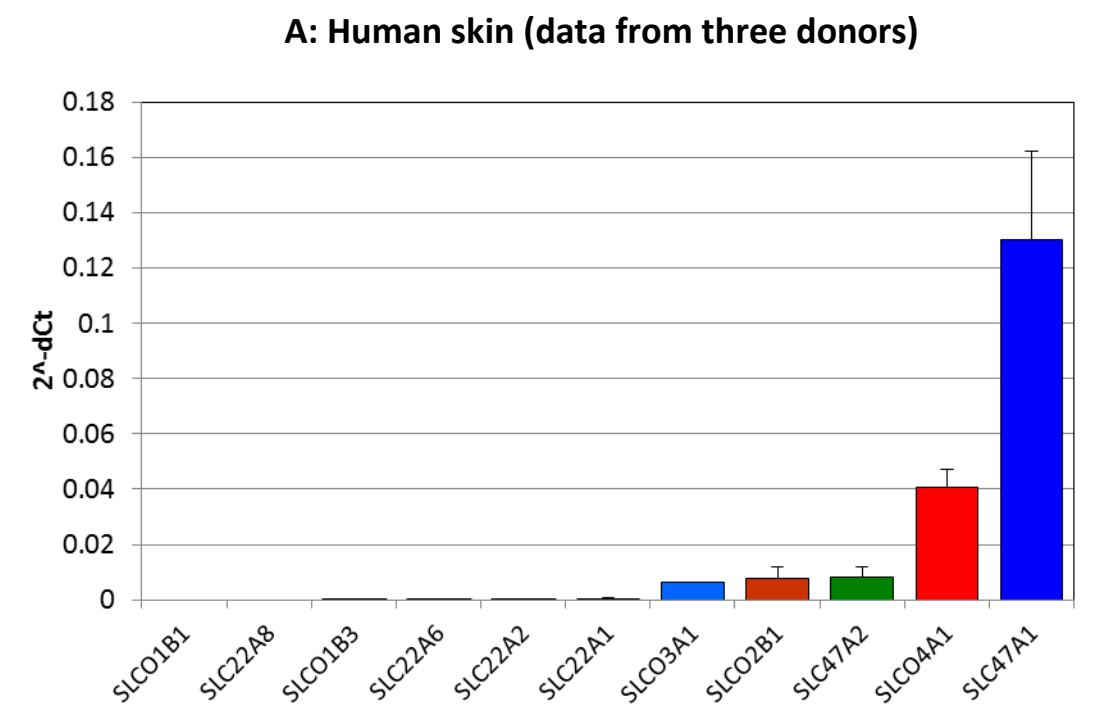

B: Human hepatocytes (Pool of 26 donors)

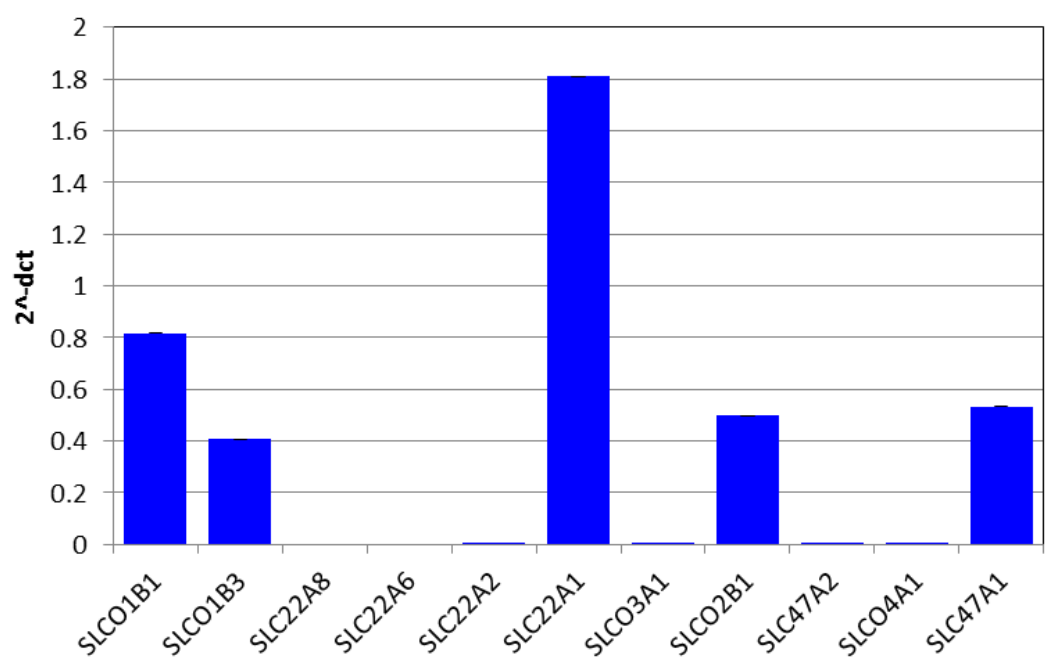

C: Human Kidney (data from 2 donors)

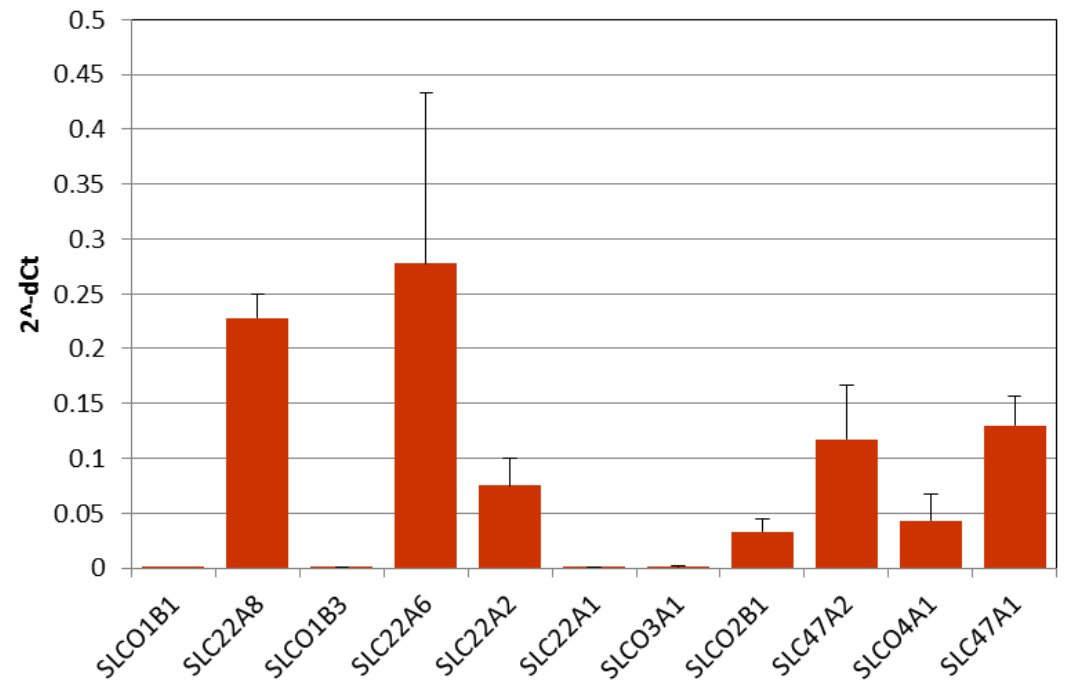

Figure 2. Constitutive expression of some SLC transporters in human skin (A), human hepatocytes (B) and human kidney (C). Quantitative real-time reverse transcription-PCR was used to investigate the constitutive expression of SLC transporters in human tissues. Gene expression was normalized to GAPDH to ensure equality of loading. All analyses were performed in triplicate. Results are means and standard error of 3 individual skin samples from 3 different donors, one hepatocyte pool of 26 donors, or 2 kidney mRNA from 2 donors. All measures were done in triplicate. 
The results presented in Figure 3 show that treatment of skin samples with rifampicin strongly decreased the expression of MATE1 and MATE2 transporters in human skin (Figure 3A; top). Indeed, rifampicin triggers a $44 \%$ decrease in MATE1 expression $(p<0.05)$ and a $30 \%$ decrease in MATE2 expression. However, treatment of skin samples with rifampicin had no effect on the expression level of OATPB, OATPD and OATPE (data not shown). The same experiment was performed with fresh human hepatocytes in primary culture treated with rifampicin $20 \mu \mathrm{M}$ for 72 hours. The results presented in Figure 3B bottom, show that the expression level of MATE1 in human hepatocytes decreased by $48 \%$ after being treated with rifampicin (data performed in triplicate on one single donor).

Overall, it means that the expression of MATE1 and MATE2 transporters in human skin is regulated by rifampicin and that the same phenomenon occurs in fresh human hepatocytes with MATE1.

\section{A: Human skin}

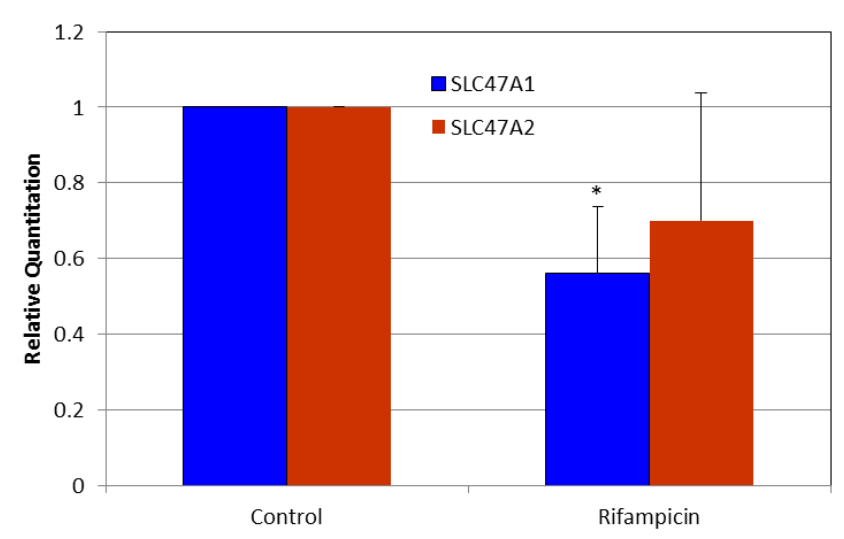

\section{B: Human hepatocytes}

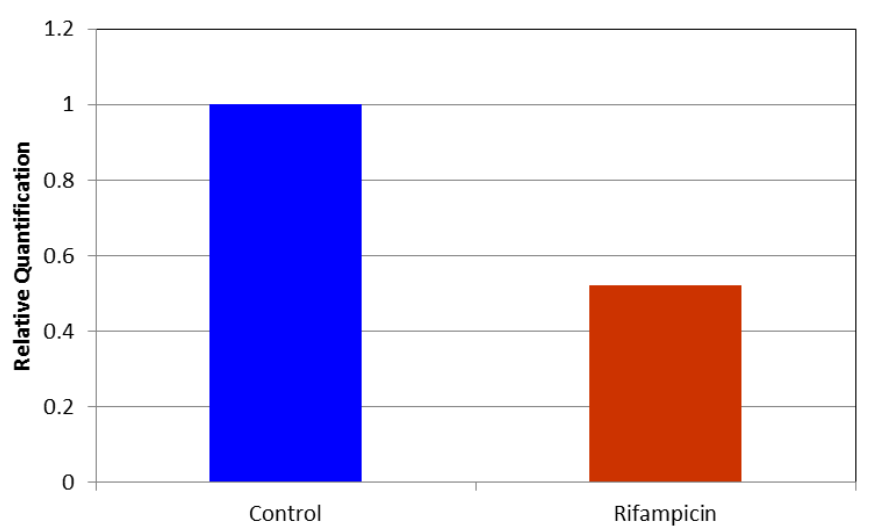

Figure 3. Effect of rifampicin on gene expression of SLC47A1 (MATE1) and SLC47A2 (MATE2) in human skin (A) and human hepatocytes (B). Human skin biopsies in organ-culture (three donors) and human hepatocytes in primary culture (one donor) were treated with rifampicin ( $20 \mu \mathrm{M})$ during 72 hours. Expression of SLC47A1 and SLC47A2 was analysed by quantitative real-time reverse transcription-PCR. Expression of mRNA was normalized to GAPDH. All analyses were performed in triplicate. Data are mean and SD of three donors (human skin). All measures were done in triplicate. ${ }^{*}$ Statistically significant $(p<$ $0.05)$.

\section{Rifampicin regulates AhR and RXRa expression in human skin}

It has been shown that the expression of ABC transporter MDR1 and MRP2 is induced by rifampicin via PXR and CAR nuclear receptors [25]. As a consequence, we investigated the involvement of nuclear receptors such as PXR, CAR, AhR and RXR $\alpha$ in regulation of SLC transporters by rifampicin. The expression 
of these nuclear receptors was measured by Real Time PCR in skin samples treated with $20 \mu \mathrm{M}$ rifampicin for 72 hours and compared to control untreated skin samples.

The constitutive expression of the four nuclear receptors in human skin, hepatocytes and kidney (Figure $4 A$, top) shows that unlike in hepatocytes, the expression of CAR and PXR was not detected in human skin (Ct above 35) and very low in kidney. However, the expression of AhR and RXR $\alpha$ was detected in human skin but at lower levels compared to hepatocytes and kidney. Moreover, expression of AhR and RXR $\alpha$ in human skin was down regulated by rifampicin, just like MATE1 and MATE2 transporters (Figure 4B; bottom). Indeed, the expression of AhR and RXR $\alpha$ decreased by $32 \%$ and $33 \%$ when skin biopsies were treated with rifampicin compared to untreated controls. This indicates that AhR and RXR $\alpha$ could be involved in MATE1 and MATE2 down regulation by rifampicin.

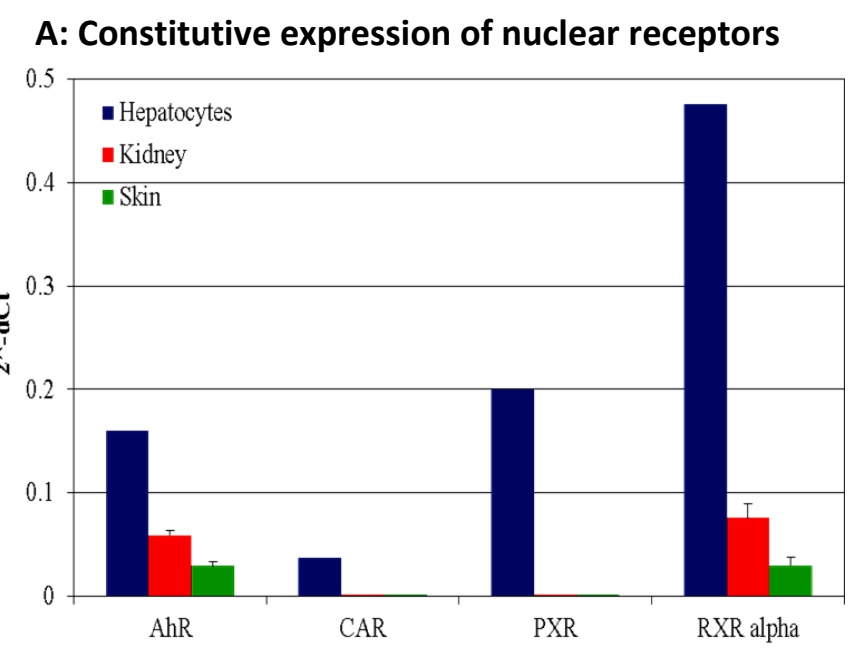

\section{B: Effect of rifampicin on nuclear receptors}

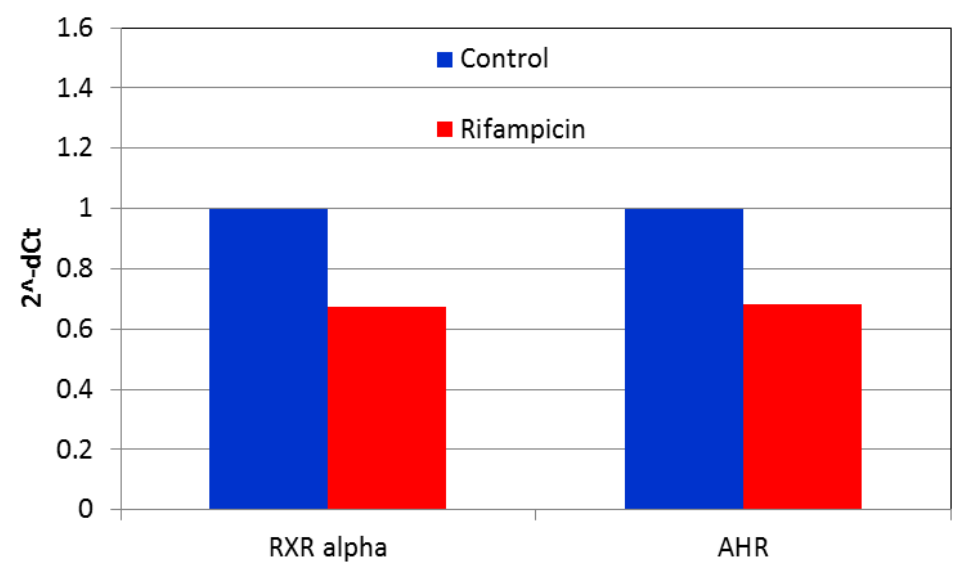

Figure 4. Constitutive expression of nuclear receptor in human hepatocytes, human kidney, and human skin (A), and effect of rifampicin on the expression of RXR $\alpha$ and AhR in human skin. (A) Results are means and standard error of 2 individual skin samples from 2 different donors, one hepatocyte pool of 26 donors, or 2 kidney mRNA from 2 donors. (B) Results from one single donor. All measures were done in triplicate.

Effect of sunlight simulator on the expression of SLC transporters in human skin

The effect of exposure to UV light on the expression of SLC transporters was investigated in two different donors. Human skin biopsies were maintained in organ-culture for three days as explained in the experimental section and exposed to UV light for 1 hour every 24 hours using a solar simulator. Control 
samples were not exposed to UV lights. The expression of the SLC transporters found to be expressed in human skin was measured by real time PCR.

The results presented in Figure 5 show that following the exposure of skin biopsies to UV light, the expression level of MATE1 and MATE2 decreased by $43 \%(p<0.05)$ and $60 \%$, respectively. The expression of SLCO3A1 and SLCO4A1 was not modified by exposure of skin biopsies to UV light (data not shown).

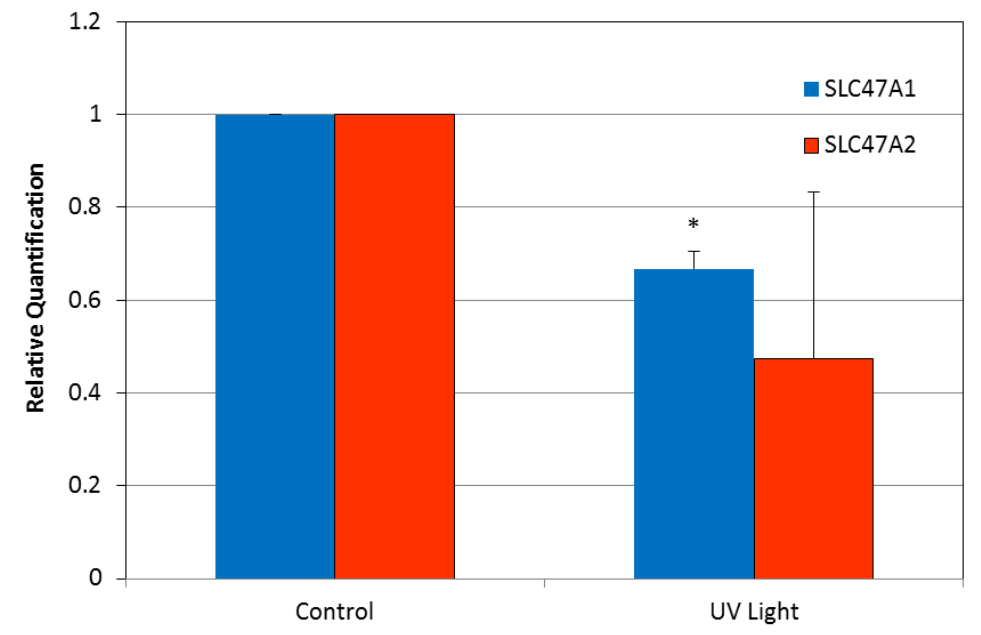

Figure5. Effect of UV light exposure on the expression of SLC47A1 and SLC47A2 in human skin. Results are means and standard error of 2 individual skin samples from 2 different donors. All measures were done in triplicate. ${ }^{*}$ Statistically significant $(p<0.05)$.

\section{Discussion}

Transporters are recognized as important actors of drug absorption, distribution and excretion. It has also been demonstrated that they are involved in drug-drug interactions, turning them into elements to be studied in drug development. ABC and SLC transporters have been well studied in liver and kidney which are responsible for most drug excretion. However, their expression and role in human skin are poorly understood. In the present study, we have observed the expression of OATPB, OATPD, OATPE, MATE1 and MATE2 transporters in human skin: OATPE and MATE1 transporters being the most highly expressed. A recent study has shown that SLC transporters were expressed in the human skin to a moderate and variable extent, but MATE transporter expression was not investigated [19]. To our knowledge it is first time that there is evidence for the expression of MATE transporter in human skin.

MATE transporters are found in many living organisms and are involved in multidrug resistance. In human, MATE1 is expressed in both liver and kidney while MATE2 is kidney-specific [26]. Those transporters play a role in renal and biliary excretion of endogenous and exogenous compounds including xenobiotics and drugs [26,27]. Although MATE1 transporter belongs to SLC family, it is an efflux transporter and its activity depends on physiological $\mathrm{pH}$. MATE1 is thought to be responsible for the last step of excretion of organic cations involving proton exchange [27]. By showing that MATE1 is strongly expressed in human skin, these data show its possible involvement in absorption, distribution and excretion of topically applied drugs. Moreover, this transporter is down regulated by rifampicin which suggests that it might play a role in drug-drug interactions. Further investigations are needed to characterize the functional role of MATE transporters in human skin. 
Furthermore, OATPE is poorly known but is highly expressed in human skin. It would be very interesting to better characterize this transporter and to investigate its role in drug disposition and to understand its function.

The results presented in this work showed that some SLC transporters have a specific expression profile in human skin. MATE1 transporter is highly expressed in human skin compared to other SLC transporters, suggesting that MATE1 may play a role in topical drug exposure and drug-drug interactions. The extensive expression of MATE1 transporter in human skin and the down-regulation by solar simulator exposure suggest that this transporter might serve as a biomarker for skin cancers, particularly those induced by solar exposure. Furthermore, the expression of MATE1 transporter in human skin may play an important role in chemosensitivity of cutaneous cancer cells and thus may be the basis for the discovery of novel agents for the treatment of skin cancers [28].

\section{Conclusions}

Our present findings suggest that OATPE and MATE1 transporters are highly expressed in human skin and that MATE1 is down-regulated by exposure to UV light, but investigations are needed to identify the potential role of OATPE and MATE1 transporters in the skin disposition of topically applied drugs.

\section{References}

[1] P.M. Elias, Current Allergy Asthma Reports, 8 (2008) 299-305.

[2] J.M. Baron, D. Holler, R. Schiffer, S. Frankenberg, M. Neis, H.F. Merck, F.K. Jugert, Journal of Investigative Dermatology, 116 (2001) 541-548.

[3] R. Schiffer, M. Neis, D. Höller, F. Rodriguez, A. Geier, C. Gartung, F. Lammert, A. Dreuw, G. ZwadloKlarwasser, H. Merck, F. Jugert, J.M. Baron Journal of Investigative Dermatology, 120 (2003) 285291.

[4] H. Schaeffer, T. Redelmeier, Skin barrier: Principles of percutaneous Absorption, S. Karger, Basel, $\mathrm{CH}, 1996$.

[5] H. Swanson, Chemico-Biological Interactions, 149, (2004) 69-79.

[6] A. Williams, Transdermal and Topical Drug Delivery, Pharmaceutical Press, London, 2003.

[7] M. Pham, J. Magdalou, M. Totis, S. Fournel-Gigleux, G. Siest, B. Hammock, Biochemical Pharmacology, 38 (1989) 2187-2194.

[8] K. Madison, Journal of Investigative Dermatology, 121 (2003) 231-241.

[9] M. Prausnitz, S. Mitragotri, R. Langer, Nature Reviews Drug Discovery, 3 (2004) 115-124.

[10] M.A. Sleeman, J.D. Watson, J.G. Murison, Journal of Investigative Dermatology, 115 (2000) 19-23.

[11] A. Dreuw, H.M. Hermanns, R. Heise, S. Joussen, F. Rodriguez, Y. Marquardt, F. Jugert, H.F. Merck, P.C. Hinrich, J.M. Baron, Journal of Investigative Dermatology, 124 (2005) 28-37.

[12] G.J. Randolph, S. Beaulieu, M. Pope, I. Sugarawa, L. Hoffman, R.M. Steinman, W.A. Muller, Proceedings of the National Academy of Sciences USA, 95 (1998) 6924-6929.

[13] R. Heise, C. Skazik, F. Rodriguez, S. Tanzel, Y. Marquardt, S. Joussen, A.F. Wendel, M. Wosnitza, H.F. Merck, J.M. Baron, Journal of Investigative Dermatology, 130 (2010) 305-308.

[14] M. Colone, A. Calcabrini, L. Toccacieli, G. Bozzuto, A. Stringaro, M. Gentile, M. Cianfriglia, A. Ciervo, M. Caraglia, A. Budillon, G. Meo, G. Arancia, A. Molinari, Journal of Investigative Dermatology, 128 (2008) 957-971.

[15] S. Heimerl, A.K. Bosserhoff, T. Langmann, J. Ecker, G. Schmitz, Melanoma Research, 17 (2007) 265273.

[16] S. Takenaka, T Itoh, R. Fujiwara, Pharmacology Research \& Perspectives 1 (2013) 1-8. 
[17] H. Osman-Ponchet, A. Boulai, M. Kouidhi, K. Sevin, M. Alriquet, A. Gaborit, B. Bertino, P. Comby, B. Ruty, Drug Metabolism and Drug Interactions, 29 (2014) 91-100.

[18] Q. Li, H. Tsuji, Y. Kato, Y. Sai, Y. Kubo, A. Tsuji, Journal of Controlled Release 110 (2006) 542-56.

[19] R. Fujiwara, S. Takenada, M. Hashimoto, T. Narawa, T, Itoh, Scientific Reports, 4 (2014) 1-8.

[20] European Medicines Agency (EMA), 2012, Guideline on the Investigation of Drug Interactions.

[21] K. Maeda, Y. Sugiyama, Molecular Aspects of Medicine 34 (2013) 711-718.

[22] US Food and Drug Administration (FDA), 2012. Guidance for Industry Drug Interaction StudiesStudy Design, Data Analysis, Implications for Dosing, and Labeling Recommendations.

[23] F.G.M. Russel, in Enzyme and Transporter-Based Drug-Drug interactions. Progress and future challenges, K.S. Pang, A.D. Rodrigues, R.M. Peter, Springer, 2010, p. 27-49.

[24] S. Haenisch, S. Laechelt, H. Bruckmueller, A. Werk, A. Noack, O. Bruhn, C. Remmler, I. Cascorbi, Molecular Pharmacology, 80 (2011) 314-320.

[25] M. Sun, W. Cui, S.K. Woody, J.L. Staudinger, Drug Metabolism and Disposition, 43 (2015) 335-43.

[26] H. Omote, M. Hiasa, T. Matsumoto, M. Otsuka, Y. Moriyama, Trends in Pharmacological Sciences, 27 (2006) 587-593.

[27] M. Otsuka, T. Matsumoto, R. Morimoto, S. Arioka, H. Omote, Y. Moriyama, Proceedings of the National Academy of Sciences USA, 102 (2005) 17923-17928.

[28] H. Osman-Ponchet, (Galderma Research \& Development), Patent WO-2014/184265 A.

(C2015 by the authors; licensee IAPC, Zagreb, Croatia. This article is an open-access article distributed under the terms and conditions of the Creative Commons Attribution license (http://creativecommons.org/licenses/by/3.0/) (cc)) EY 